\title{
The effect of stimulus complexity on retrieval of information from memory
}

\author{
PHILIP TOLIN* and GEORGE M. DELEGANS \\ Central Washington State College, Ellensburg, Washington 98926
}

\begin{abstract}
Eighteen Ss performed an item recognition task involving memorized lists of one, two, and three nonsense forms at one of two levels of stimulus complexity. Analysis of RT-set size functions and serial position functions suggested that (a) when the memorized list consisted of simple figures, Ss engaged in a serial exhaustive search of items stored in memory prior to responding and (b) when the memorized items were complex, an increase in set size was accompanied by a change in search strategy from a backward self-terminating search to a random self-terminating search.
\end{abstract}

In recent years, there has been renewed interest in studying memory storage and retrieval processes in the context of recognition memory experiments. In a typical recognition memory task, a brief list of items is presented for memorization. The $S$ is then asked whether a test, or probe, item was contained in the memorized list; $\mathrm{S}$ responds as quickly as possible and his reaction time (RT) is measured. Inferences concerning underlying memory processes are typically based on the relationships between RT and (a) the size of the memorized list and, (b) on trials on which a positive response is demanded, the serial position of the probe item in the memorized list.

Several item recognition studies have shown a linear relationship between mean RT and the size of the memorized, or positive, set of stimuli (Haber, 1969; Neisser, 1967; Olshavsky \& Gregg, 1970; Standing \& Haber, 1968; Sternberg, 1966, 1967, 1969). These results suggest that Ss engage in a serial search process to determine whether an agreement exists between the probe and any of the items in the positive set.

Two types of serial search have been suggested (Sternberg, 1969): exhaustive and self-terminating. In an exhaustive search, even though the probe may match a member of the positive set, the $\mathrm{S}$ does not respond until all positive stimuli in memory have been evaluated. Thus, it would be expected that the rate at which RT increases with set size will be the same for positive and negative responses. Additionally, serial position functions (mean RT of positive responses as a function of serial position of items in the positive set) should be flat, since all items are searched before a response is made.

In a self-terminating search, the comparison process continues only until a match occurs. On the average, the search terminates in the middle of the list for positive probes but continues through the entire list following presentation of a negative probe. Thus, there should be a Probe Type by Set Size interaction in which the rate at which RT increases with set size should be twice as great

*Address: Department of Psychology, Central Washington State College, Ellensburg, Washington 98926. for negative responses as for responses to positive probes. A self-terminating search that began with the first-presented item and proceeded serially would result in an increasing serial position function (Sternberg, 1969); a backward self-terminating search would result in a decreasing serial position function (Clifton \& Birenbaum, 1970). A random self-terminating search would produce a flat serial position function.

Several types of stimuli, including digits, letters, nonsense forms, and photographs of faces, have been used in such recognition memory experiments. Data have suggested that $S$ s engage in both exhaustive search processes (e.g., Standing \& Haber, 1968; Sternberg, 1969) and self-terminating processes (e.g., Clifton \& Birenbaum, 1970; Corballis, 1967; Klatzky \& Atkinson, 1970). The effects of variations in several stimulus parameters on underlying retrieval processes have not been investigated. The purpose of the present study was to investigate the effects of one of these parameters, stimulus complexity.

\section{METHOD}

\section{Subjects}

The Ss were 18 naive male volunteers from an introductory psychology class. All reported normal or corrected-to-normal vision.

\section{Apparatus}

Each $\mathrm{S}$ was seated in a sound-attenuated room, $2 \mathrm{ft}$ in front of a rear-projection screen placed in front of a window. Stimuli were sequentially projected on the screen at eye level by a Kodak carousel random-access projector located in an adjacent room.

The stimuli were 32 random shapes, 16 at each of two levels of complexity, taken from Vanderplas and Garvin (1959). The "simple" shapes had four points of inflection on their perimeters, while the "complex" shapes had eight such points. The two sets of stimuli were matched in terms of association value. From each ensemble of 16 shapes, 6 were randomly selected to constitute the three positive sets (set sizes $=1,2$, or 3 ). The remaining 10 shapes in each ensemble constituted the negative probes. 


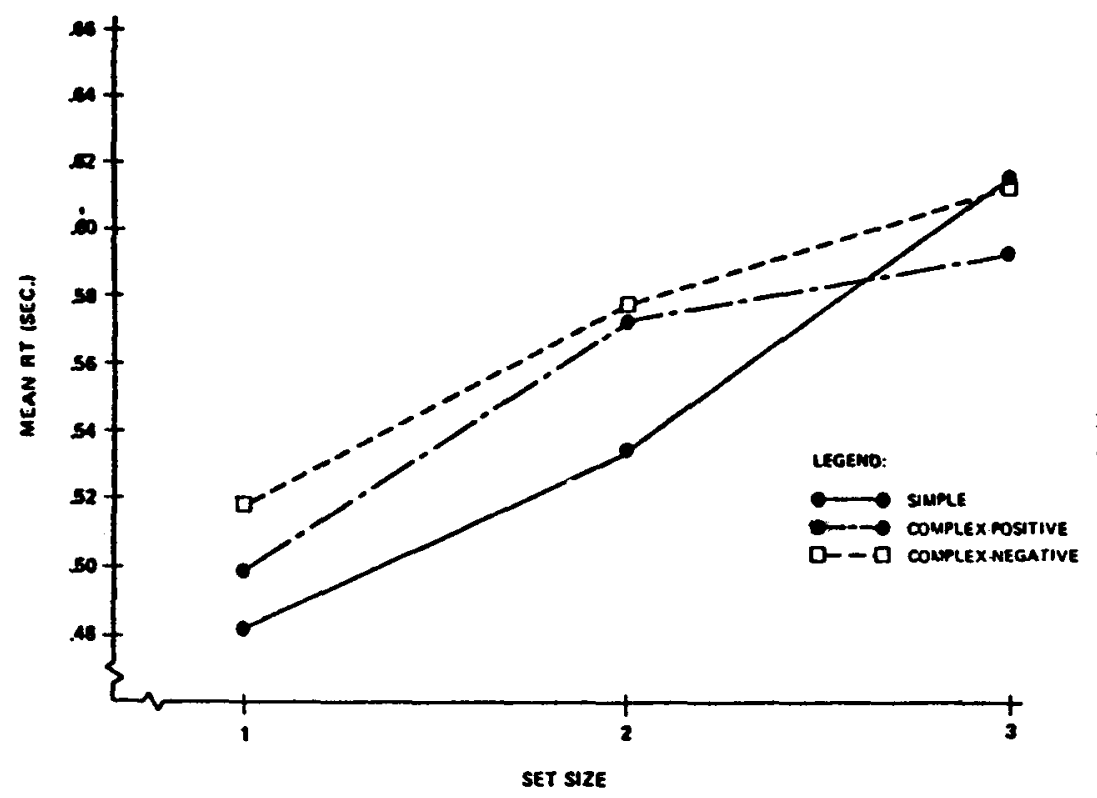

Fig. 1. Mean RT as a function of set size at both levels of stimulus complexity. Reaction times of responses to positive and negative probes are combined for the simple condition.

\section{Design and Procedure}

The Ss were assigned randomly to one of the two levels of stimulus complexity and tested with all three set sizes on consecutive days in a counterbalanced arrangement.

A fixed-set procedure (Sternberg, 1969) was used: Within each set size condition, the positive set remained constant from trial to trial. Each trial began with the serial presentation of the positive set. Stimulus duration and interstimulus interval were each $1 \mathrm{sec}$. Two seconds after the last shape in the positive set was displayed, a probe was presented. Figures in the memorized list were presented on clear backgrounds, while probes were presented on a light orange background. The intertrial intervals were $2 \mathrm{sec}$.

Probes were selected so that positive responses were correct on a random half of the trials. The positive probes were distributed equally across serial positions. The Ss were instructed to respond as rapidly as possible following presentation of the probe by pressing one button if the probe was identical to any member of the positive set (positive probe) and another button if the probe was not a member of the positive set (negative probe). Half of the Ss made positive responses with the preferred hand and negative responses with the nonpreferred hand; the response pattern was reversed for the remaining $S$ s.

To minimize error rates, each $\mathrm{S}$ was given a practice session that continued to a criterion of eight successive errorless trials. All stimuli were the same for both practice and experimental sessions. The experimental session, which immediately followed the practice trials, ran for 120 trials. This procedure was repeated for each of the three set sizes.

\section{RESULTS}

An overall analysis of the main and interactive effects of stimulus complexity, set size, and probe type (positive or negative) on RTs was followed by separate analyses at each level of stimulus complexity. Effects of serial position of positive probes on RTs were also evaluated. Reaction times of correct responses only were analyzed; error rates were approximately $2 \%$.

\section{Reaction Time/Set Size Relationships}

The overall analysis indicated that stimulus complexity did not exert a significant main effect on RT $(p>.05)$. The effect of set size was significant $[F(2,32)=59.00, p<.01]$, and there was a significant Stimulus Complexity by Set Size interaction $[F(2,32)=4.00, p<.05]$. These effects are illustrated in Fig. 1.

In Fig. 1 the $\mathrm{RT} / \mathrm{set}$ size function for the simple condition has been collapsed across the two probe types, since for these slides probe type did not exert a significant main or interactive effect on RT, i.e., there was no statistical difference between the functions for positive and negative responses. Curves for responses to positive and negative probes are shown separately for the complex condition, however; while the main effect of probe type was not significant $(p>.05)$, there was a significant Probe Type by Set Size interaction $[F(2,40)=5.00, p<.05]$. It can be seen in Fig. 1 that an increase in set size from 1 to 2 had comparable effects on positive and negative responses; the increase in set size from 2 to 3 , however, had a more pronounced effect on RTs to negative probes. The latter increase in mean RT to negative probes was 1.74 times as great as the corresponding inorease in RTs to the positive probes.

Trend analyses indicated that, for simple figures, departures from linearity in the RT/set size function were not significant $(p>.10)$; the function may be considered statistically linear. For the complex figures, however, there was a significant quadratic component in the $\mathrm{RT} /$ set size function for responses to positive probes $[F(1,40)=4.30, \quad p<.05]$ but not for negative responses.

\section{Serial Position Functions}

With one exception, serial position effects were not significant $(p>.10)$, i.e., serial position functions were essentially flat. The exception was the serial position function for complex stimuli at set size $=2$. This 
function showed a pronounced recency effect $[F(1,16)=10.50, p<.01]$, i.e., probes corresponding to the second item in the positive set elicited more rapid responses than probes corresponding to the first item in the positive set.

\section{DISCUSSION}

The finding of a linear relationship between RT and set size for simple figures is consistent with the results reported previously. This linear trend, and the absence of a significant interaction between set size and probe type, suggest that with simple figures Ss engaged in a serial exhaustive search (Sternberg, 1969) of items stored in memory prior to responding. Additional support for this assumption is provided by the statistically flat serial position functions.

The data suggest, however, that a more complex conception of the memory scanning process, involving self-terminating search strategies, is warranted when more complex stimuli are stored in memory. It will be recalled that for these items there was a significant Set Size by Probe Type interaction. Examination of the interaction reveals that the effect of increasing set size from 1 to 2 was similar for both positive and negative responses; the functions are essentially parallel over this range. However, the effects of increasing set size from 2 to 3 were clearly dissimilar for positive and negative responses; the increase in RTs of negative responses was greater than the increase for positive responses. The magnitude of the difference in rate of increase in RT closely approximates that suggested by Sternberg (1969) in the case of a self-terminating search.

This divergence between the latter two points, coupled with the absence of a serial position effect at Set Size 3, suggests that when the memory load was sufficiently great $S s$ engaged in a random self-terminating search (Sternberg, 1969). At Set Size 2, however, the obtained serial position function suggests that Ss engaged in a backward self-terminating search.

It is possible to speculate about factors underlying this suggested change in search strategies in terms of Sternberg's (1969) memory scanning model. According to this model, the slope of the RT/set size function is a measure of the rate of the scanning process. The zero intercept is presumed to be a measure of the time taken by processes occurring just once-regardless of set size-particularly, the encoding of stimuli to form their representations in memory.

Because of the departure from linearity of the RT/set size function for complex stimuli (Fig. 1), it is necessary to consider this function in two segments, across Set Sizes 1 and 2 and across Set Sizes 2 and 3. The difference in slopes of these two segments is evident and suggests that scanning rate is faster for the largest set size than for the smaller sets. With set size $=3$, the scanning process appears to have been more efficient than at Set Sizes 1 and 2.

Extrapolation of these segments to the zero intercept suggests a large difference in encoding time, with more time taken by this process for large set sizes. It is suggested that the greater time taken by the encoding process facilitated the process of retrieval of stored information. Possibly, this difference in encoding time, if real, is related to the perceived level of task difficulty. If so, encoding and retrieval processes should be affected by manipulation of extrastimulus variables, e.g., instructions.

\section{REFERENCES}

Clifton, C., \& Birenbaum, S. Effects of serial position and delay of probe in a memory scan task. Journal of Experimental Psychology, 1970, 86, 69-76.

Corballis, M. C. Serial order in recognition and tecall. Journal of Experimental Psychology, 1967, 74, 99-105.

Haber, R. N. Information processing analyses of visual perception: An introduction. In R. N. Haber (Ed.), Information processing approaches to visual perception. New York: Holt, Rinehart, \& Winston, 1969.

Klatzky, R. L., \& Atkinson, R. C. Memory scans based on alternative test stimulus representations. Perception \& Psychophysics, 1970, 8, 113-117.

Neisser, U. Cognitive psychology. New York: Appleton-Century-Crofts, 1967.

Olshavsky, R. W., \& Gregg, L. W. Information processing rates and task complexity. Journal of Experimental Psychology, $1970,83,131-135$.

Standing, L., \& Haber, R. N. Visual search and memory under degraded and masked presentation. Psychonomic Science, 1968, 13, 81-82.

Sternberg, S. High-speed scanning in human memory. Science, $1966,153,652-654$.

Sternberg, S. Retrieval of contextual information from memory. Psychonomic Science, 1967, 8, 55-56.

Sternberg, S. Memory scanning: Mental processes revealed by reaction time experiments. American Scientist, 1969, 57, 421-457.

Vanderplas, J. M., \& Garvin, E. A. The association value of random shapes. Journal of Experimental Psychology, 1959, $57,147-154$.

(Received for publication April 3, 1973; accepted April 7, 1973.) 